(Aus dem Pathologischen Institut zu Genf.)

\title{
Bedeutung der Membrana propria der Glomerulusschlingen in der Nierenpathologie.
}

\author{
Von
}

\author{
Dr. med. D. Ohmori.
}

(Eingegangen am 6. April 1921.)

Die normale und pathologische Histologie sowie die experimentelle Pathologie haben miteinander gewetteifert, um den feineren Bau der Niere möglichst gründlich klarzulegen. Die immer lebendige und heute besonders gepflegte Forschung über die physiologische Funktion der Niere und ihrer Teile hat besonders an die experimentelle Nephritis, ferner an die Ausscheidung körperfremder Stoffe angeknüpft. Vielleicht ist das interessanteste, demnach am eifrigsren studierte Gebiet das von der Rolle der Malpighischen Körperchen. Die Meinungen über, Nierenkörperchen und Harnkanälchen in der normalen Histologie Physiologie und Pathologie sind heute schon ziemlich einstimmig, aber es gibt doch natürlich einiges, was weitere Untersuchungen verlangt, ja. was bisher vernachlässigt ist.

Der feinere Bau des Glomerulus ist vielfach untersucht. Wie auch Gross jüngst wieder in seiner Abhandlung erwähnt hat, sind die Meinungen der Histologen und Pathologen noch weit von einer gemeinsamen Anschauung entfernt. Wenn v. Ebner auch an der Wand der Knäuelschlingen die Auskleidung der Endothelien leugnete und sie nur als eine Art zusammenhängender Protoplasmamasse ohne Kerne. darstellte und auch bekanntlich v. Hansemann und Ribbert ein Glomerulusendothel nicht anerkannt haben, werden von Langhans, Löhlein und anderen die Schicksale der Endothelien der Glomerulusschlinge, ihre Vermehrung und Vergrößerung als Ausgangspunkt der histologischen Veränderungen bei der Glomerulonephritis betrachtet. Gross sagt: In den Arbeiten der letzten Zeit (Fahr, Herxheimer) wird die Frage gar nicht mehr erörtert, sondern das Vorhandensein allerdings spärlicher Endothelkerne und ihre Vermehrung. bei der Glomerulitis nur als bekannte Tatsache erwähnt. Dasselbe gilt auch von der kernreichen Masse, die außerhalb der Knäuelschlingen vorhanden ist. Einerseits stellen die Histologen, wie v: Ebner, Schultze und andere, diese als Syncytium hin; andererseits schildern fast alle Pathologen diese einfach als Epithelien. Löhlein hat sie in 
seinen Abhandlungen als „Knäuelepithelien“, Herxheimer als „Schlingenepithelien" beschrieben. Aber Gross will sie mit Rücksicht auf die Kerne, die er häufig in besonderer Form in der Mitte des Knäuelkonvolut gefunden hat, nicht als Epithelien ansehen und die ganze kernreiche Masse als „Knäuelüberzug“ bezeichnen. Außerdem wird das Vorhandensein von Bindegewebe in den Glomerulusknäueln noch exörtert, im allgemeinen aber abgelehnt.

Alle diese bisher genannten Punkte sollen hier nur nebenher gestreift werden, wo wir einen anderen Teil am Glomeruluskörper ins Auge zu fassen wünschen. Es ist eigenartig, daß fast alle Autoren bei diesen histologischen Untersuchungen über Glomeruli kein Wort für die Membrana propria der Glomerulusschlingen übrig haben. Nur Langhans hat, und zwar infolge gewisser pathologiseher Befunde, an den Glomerulusschlingen außer diesen Elementen noch eine Membrana propria, die das Endothelrohr der Glomerulusschlinge umhüllt, unterschieden, während Hansemann dagegen nur von einer „einfachen Wand" der Glomeruluscapillaren spricht. Später haben aber Hueter und Goldschmid wieder von dieser Membrana propria eindeutig gesprochen. In den meisten Abhandlungen haben aber die Autoren im allgemeinen einfach von dem Zustand der Schlingen-, Capillar- oder Knäuelwand gesprochen (z. B. als verdickt, breit, starr usw.) und sich dabei hauptsächlich mit Endothelien und Epithelien der Glomurulusschlingen beschäftigt (Löhlein, Herxheimer, Fahr, Gross und andere). Indessen scheint Löhlein zuweilen als Schlingenwand im engeren Sinne die Membrana propria im Auge gehabt zu haben, und Gross dürfte auch einmal das Wort „Grenzmembran" wahrscheinlich für die Membrana propria angewendet haben, aber wie erwähnt, wird die Membran nur oberflächlich gestreift. Ja gilt es schon als Frage, ob die Membrana propria der Glomerulusschlingen wirklich vorhanden ist oder nicht.

Wenn im Glomerulusknäuel außer Endothelien und Epithelien noch eine besondere Wand, und zwar als Membrana propria vorhanden ist, muß sie auch in der physiologischen Funktion und in der Pathologie des Glomerulus eine gewisse Rolle spielen. Wie sie an der Funktion des Glomerulus teilnimmt, oder ob sie eine wesentliche Bedeutung hat, ist eine weitere Frage. Noch wichtiger könnte ihre Rolle bei verschiedenen Erkrankungen der Glomeruli, die man doch heute viel höher einschätzt als früher, ein nicht zu vernachlässigendes Problem sein. Ihr Schicksal bei verschiedenen Glomeruluserkrankungen zu verfolgen, ist eine Aufgabe, an die ich auf Anregung von Herrn Prof. Askanazy heranging. Denn es kann für jeden, der sich einmal die Mühe genommen hat, Nieren einige oder selbst längere Zeit post mortem frisch zu untersuchen, kein Zweifel bestehen, daß nach dem Untergang oder der Ablösung 
der zelligen Elemente im Glomerulus ein Gebilde übrigbleibt, das noch völlig die Gestalt der Gefäßknäuel bewahrt. Dann ist es nun eine Art von zellfreiem Glomerulusskelett, das die Membrana propria in reiner Form darstellt. Ja man kann bemerken, daß die membranösen Röhrchen an diesen Glomeruli schärfer hervortreten als während ihres vitalen Zustandes. Vielleicht kommt es bei der Fäulnis zu leichten, Quellungen dieser Membrana propria, die sie anschaulicher gestaltet. Natürlich braucht man für genauere Studien feine Schritte und geeignete Färbungsmethoden.

Vor der Beschreibung unserer Beobachtungen wollen wir daher kurz von der Färbung der Membrana propria sprechen, die natürlich von den Zell- und Kernfärbungen nicht dargestellt wird. Um diese Membran tinktoriell in exakter Weise zur Anschauung zu bringen, kommen Methoden zur Darstellung von Grundsubstanzen in Betracht. Außer gewöhnlichen Hämatoxylin-; Eosin- und v. Gieson-Färbungen haben wir für unseren Zweck die Silberimprägnationsmethode nach Bielschowsky-Maresch probiert, die ja oft zum Studium von Grundsubstanzen verwertet ist. Mit Eosin färbt sich die Membran zu blaß, und an den dünnsten Schnitten ist es fast unmöglich, sie zu erkennen. Bei $\nabla$. Gieson-Färbung gelingt die Färbung besser, aber oft auch noch nicht recht prägnant; etwas bessere Resultate gewinnt man durch reicheren Zusatz von S-Fuchsin. Die Ergebnisse der Silberimprägnation waren nicht gleichmäßig. Die Tunica propria der Harnkanälchen war meist schön schwarz gefärbt und unterscheidet sich gut von dem mehr rötlich gefärbten interstitiellen Bindegewebe. Allein für das Studium der Membrana propria der Glomeruli waren die Resultate zu launisch, die Färbung nur streckenweise, oft unscharf, und es gelang nur, in kleineren Strichen, diese Membran als Schwarzlinie anschaulich zu machen.

Wir haben mehr Erfolge erzielt mit einer Methode, die man Herrn Prof. Askanazy verdankt, der schon lange Reinblau für diesen Zweck anwendet. Nach seiner Vorschrift wird Reinblau (doppelt konzentriert) mit Pikrinsäure gemischt, um zugleich die acidophilen Zellsubstanzen und Erythrocyten zu färben. Damit ist die Vorfärbung der Kerne zu kombinieren. Für die Kernfärbung haben wir Lithionkarmin oder Säurefuchsin, besonders das letztere, angewendet. Zur Herstellung der genannten Farblösung setzt man zu einer konzentrierten wässerigen Pikrinsäurelösung so viel von einer stärkeren wässerigen Reinblaulösung, daß eine dunkelblaugrüne Lösung resultiert. Man braucht nach der Kernvorfärbung die Schnitte nur eine Minute in dieser grünen Lösung zu färben, differenziert dann in Alkohol (evtl. mit Pikrinsäurezusatz) und behandelt wie üblich weiter. Durch diese Färbung haben wir in der Tat vortreffliche Bilder bekommen. Die Membrana propria der Glomerulusschlingen stellt sich dabei schön blau gefärbt dar und 
tritt so deutlich hervor, daß man ihren geschlängelten Verlauf distinkterweise verfolgen kann und infolgedessen die Unterscheidung zwischen extra- und intracapillär sitzenden, von Säurefuchsin leuchtend rot gefärbten Kernen meist nicht schwer fällt. Außerdem wird die Tunica. propria der Harnkanälchen tiefblau gefärbt, und die anderen Gewebsteile sind im allgemeinen blaßblaugrünlich. Es ist dabei noch interessant, daß die Granula in den Epithelien der Harnkanälchen (veränderte Altmannsche Granula) auch feuerrot gefärbt deutlich erscheinen.

Für die Färbung dieser Membrana propria hat Hueter außer den verschiedenen Methoden der Fibrinfärbung auch die von Ribbert angegebene Methode der Bindegewebsfärbung mit Phosphormolybdänsäure, Mallorys Hämatoxylin und auch die Mallorysche Methode mit Säurefuchsin-Anilinblau-Orange hervorgehoben. Außerdem hat er auch betont, daß bis zu gewissen Grade veraltetes Weigertsches Fuchselin zu diesem Zweck brauchbar ist, welches zur Darstellung des elastischen Gewebes nicht mehr brauchbar, aber doch auch nicht zu alt ist. Gross benutzte auch Cresylviolettfärbung, um die Grenzmembran in Glomerulus deutlich hervortreten zu lassen und eine gute Unterscheidung der Infiltratzellen zu ermöglichen. Wir haben keine Gelegenheit gehabt, alle diese verschiedenen Methoden zu probieren. deren Vielheit schon eine bedenkliche Sprache redet. Unsere Methoden gaben befriedigende Resultate.

Wir werden im folgenden unsere Befunde im einzelnen schildern, die an 10 Fällen erhoben sind, bei denen die Membrana propria beachtet werden muß: 1 Fall von Argyrie, 4 Amyloidnieren und 5 Fälle von Glomerulonephritiden. Natürlich wird dabei der Glomerulus, besonders die Wand der Capillarschlingen, hauptsächlich in Betracht gezogen, während die histologischen Befunde der anderen Gewebe nur kurze Erwähnung finden, soweit sie selbst noch Interesse für die. Beurteilung bieten.

Fall 1. Argyrosis renum. Älteres Präparat des Genfer Museums.

In diesem Falle ist die Mehrzahl der Glomeruli noch wohl erhalten, abgesehen von leichten postmortalen Veränderungen, die die Lösung von Epithelien und Verblassung der Kernfärbung an einzelnen Stellen bedingten. Andere Glomeruli aber sind erkrankt, und zwar oft obliteriert. Von solchen veränderten Glomerulis finden sich mehrere, ca. ein Dutzend Exemplare in jedem Schnitte. Im allgemeinen treten die Zellelemente im Bereiche des Glomerulus noch besser vor als das Kapselepithel, an dem hie und da eine Zelle mit genügender Kern- und Protoplasmafärbung hervortritt. Diese Zellen liegen mehrfach noch der Bowmanschen. Membran an, welche hie und da etwas hyalin und verdickt erscheint. Manchmal haben die Kapselepithelien sich abgelöst. Am Gefäßknäuel sind die Epithelien, welche die Schlinge bekleiden, oft gut zu unterscheiden. Im Inneren des Capillarlumens ist meist keine Zelle zu sehen. Nicht ganz selten treten aber doch kernhaltige Zellen hervor, die teils Blutzellen (Lympho- und Leukocyten), teils platte Zellen mit bläschenförmigen Kernen darstellen. Sie haben, wenn sie mitten in den 
ziemlich weiten Capillarlumen auf oder neben der Schlingenwand hervortreten, ganz das Aussehen von Endothelien. Nur bei Schlingen, die nahe ihrer oberflächlichen Wölbung angeschnitten sind, können Zweifel zwischen Capillarendothel und Glomerulusepithel entstehen. Die Endothelzellen bilden jedenfalls keine geschlossene Zellage, was schon mit Rücksicht auf das Alter des Präparates auch an anderen Gefäßen nicht zu erwarten war. Auffallend war in den Präparaten, deren Kerne mit Säurefuchsin gefärbt sind, daß die Endothelkerne dunklere Rotfärbung zeigen als die Epithelkerne.

Am interessantesten ist aber, daß die Glomeruli ohne Ausnahme durch die schwarze Pigmentierung von Silberkörnchen ausgezeichnet sind. Die Körnchen. sind recht klein, aber untereinander von ziemlich gleicher Größe. Eine wechselnde Stärke der Pigmentierung der Glomeruli ist nur dadurch bedingt, daß die mehr oder weniger veränderten Knäuel wegen der dichten Lagerung im zusammengedrïckten Abschnitt der Silberkörnchen schwarz gefärbt sind. An allen noch normale Konfiguration darbietenden Glomeruli, deren Schlingen also noch durchgängig sind, zeigt sich nun die Silberablagerung in konstanter Weise folgendermaßen: In der Bowmannsehen Membran liegen die Silberkörnchen nur spärlich und gern an der Stelle, wo das Vas afferens in den Glomerulus eintritt. Seltener kann von hier aus die Imprägnation von Silberkörnchen sich etwas weiter in der Kapselmembran ausdehnen. Oft ist die ganze Bowmansche Membran vollkommen pigmentlos. Das Kapselepithel enthält ebensowenig Pigmentkörnchen wie das Glomerulusepithel. Überall an der Oberfläche des Knäuels und zwischen den Schlingen sind, die unpigmentierten Epithelzellen deutlich von der pigmentierten Schlingenwand abzugrenzen. Nicht anders steht es mit den Zellen, die innerhalb der Glomeruluscapillaren vorhanden sind: alle Zellelemente, Leukocyten und Endothelien erscheinen frei von schwarzen Körnchen. Nur in der Nachbarschaft der Endothelkerne sind hie und da wenige schwarze Körnchen sichtbar. Es sieht aber nur selten so aus, als gehörten die Körnchen zu ihrem Protoplasma. Dagegen zeigt sich das ganze Konvolut der Schlingen in gleichmäßiger Weise von einer schwarz pigmentierten Membran gebildet, die schon nach ihrer Lage nicht anders sein kann als die Membrana propria. Man kann in der Tat alle Windungen und Faltungen der Membran an dem schwarz pigmentierten Häutchen wiederfinden. Die v. Giesonfärbung lassen Bowmansche Kapsel und Tunica propria der Harnkanälchen gut hervortreten, aber nicht die Grundmembran der Glomeruli, weil die Silberkörnchen zu dicht in ihr eingelagert sind. In Reinblaufärbung kann man an dünnsten Stellen nur noch einen blauen Hauch als Grundlage der schwarz pigmentierten Röhrchen erkennen, da die Membran zu dicht von dem Pigment durchsetzt ist.

Wenn wir uns zu den in Verödung begriffenen Glomeruli wenden, so läßt sich mit Leichtigkeit feststellen: 1. die teilweise deutlich schwarz pigmentierte Kreislinie der Bowmannschen Kapsel, die sich an manchen Glomeruli fast in der ganzen Circumferenz verfolgen läßt, 2. eine breite fibröse, in v. Giesonfärbung rot gefärbte Kugel, die im Zentrum ausgehöhlt ist und 3. endlich innerhalb derselben der Rest der Glomerulusschlingen. In der fibrösen Zone erkennt man sklerotisches Bindegewebe mit spärlichen spindelförmigen Kernen, in dem Silberkörnchen nicht hervortreten. In der dritten Zone wechselt das Bild des verödeten Glomerulusknäuels von einer Stelle zur anderen. Bald erkennt man noch die zusammengepreßten, von Silberkörnchen durchsetzten Röhrchen mit dem komprimierten Lumen, bald ist nur noch eine schwarz pigmentierte Masse übrig geblieben, deren Konturen außen unregelmäßig, beinahe zackig erscheinen. Meist erkennt man aber an der Außenfläche der Kugel noch einen welligen Kontur. An den Stellen, wo einzelne Schlingen noch erkennbar sind, liegen auch sehwarze 
Körnchen in der Membrana propria distinkt verteilt. Da, wo die Konfiguration des Glomerulus ausgelöscht ist, ist stellenweise nur eine dunkle, schwarze Masse sichtbar, die aber an einzelnen Punkten immer noch die schwarzen Körnchen. erkennen läßt. An manchen Glomerulusdurchschnitten sieht man, daß die fibröse, die schwarzen Schlingen umgebende Kugel mit dem perikapsulären Bindegewebe in Verbindung steht. An solchen Stellen ist dann die Bowmansche Membran unterbrochen. In allen komprimierten schwarzen Glomeruli, die von bindegewebigen Hüllen umsohlossen sind, sind Kerne nicht mehr zu erkennen.

Die Harnkanälchen in der Rinde sind nicht wesentlich verändert. Ihre Tunica propria zeigt auch keine Silberimprägnierung. Aber in den Pyramiden verhält es sich ganz anders. Hier sind fast alle Tubuli recti dadurch ausgezeichnet, daß ihre Tunica propriae mit schwarzen Körnchen besetzt sind. Doch sind die Silberkörnchen hier keineswegs so dicht wie in den Glomeruli. Hier ist die Silberpigmentierung bei schwacher Vergrößerung eben noch sichtbar, während die Schwarzfärbung der Glomeruli sofort in die Augen sticht. Man sieht diese Pigmentierung sowohl an den ab- und aufsteigenden Schenkeln der Henleschen Schleifen als auch an den Sammelröhren, wo die Pigmentierung oft etwas blasser ist. Da das Bindegewebe in den Pyramiden gewuchert ist, wären die Tunica propriae sehr schwer abzugrenzen, wenn sie nicht durch die Silberpigmentierung gekennzeichnet wären. In einzelnen Gruppen von Kanälchen findet man ziemlich zahlreiche hyaline Zylinder. Interessant ist das Vorhandensein von Silberniederschlägen an einzelnen Stellen, wo das Bindegewebe der Pyramiden stark sklerosiert ist und anscheinend ganz atrophierte Kanälchen eingeschlossen liegen. Das interstitielle Bindegewebe der Rinde ist auch mäßig verbreitert, im ganzen aber kernarm. Die Arterien, welche zu der Rinde aufsteigen, sind offenbar mehrfach in starker Sklerose mit Neubildung oder Zerlegung der alten Elastica interna in mehreren Lamellen. Die Venen sind ziemlich stark erweitert.

Zusammenfassung: Die Argyrie spricht sich bekanntlich in Niederschlägen gleichmäßig feiner Silberkörnchen aus. Am Schnitte sieht man, wie das makroskopische Bild erwarten läßt, Silberniederschläge hauptsächlich in der Rinde, die in allererster Linie, ja fast ausschließlich, an die Glomeruli gebunden sind, und dann in den Pyramiden. Es ist interessant, gerade an den Glomeruli zu beobachten, welche Beziehungen zwischen diesen Silberkörnchen und den Glomeruluselementen bestehen und welches Schicksal die durch Versilberung gestempelten Schlingen bei der Obliteration erfahren. Dabei nehmen wir das Vorhandensein der Membrana propria an den Glomerulusschlingen durch ihre Imprägnierung mit Silberkörnchen und das Vorhandensein der Schlingenendothelien innerhalb dieser Membran wahr. Alle Erwägungen und Beobachtungen sprechen dafür, daß das auf dem Blutwege herankommende gelöste Silber in den Membranae propriae der Glomerulusschlingen in feinster, körniger Form niedergeschlagen wird. Daraus ist zu schließen, daß die Grundmembran des Glomerulus in elektiver Weise diese Silberkörnchen aus dem vorbeiströmenden Blut aufnimmt. Interessant ist, daß die Endothelzellen der Glomerulusschlingen an dieser Stoffaussonderung aus der Zirkulation keinen Anteil nehmen. Bei der Verödung des Glomerulus, die 
ihrerseits unabhängig von der Argyrie ist, bleibt die Membrana propria der Knäuelschlingen am längsten nachweisbar, noch lange wird ihr Kontur durch die Silberdurchtränkung deutlich gezeichnet, selbst nach dem Verschwinden aller zelligen Elemente des Glomerulus. Endlich kann man erkennen, daß in der von außen durch die Glomeruluskapsel eingedrungenen Bindegewebemasse noch ein Einschluß steckt, der aus der verbackenen Masse der Membrana propria hervorgegangen ist.

Wir betrachten nun 4 Fälle von Amyloidnieren, deren Diagnose natürlich durch Amyloidfärbung nebenbei gesichert wurde.

Fall 2. Amyloidniere.

In diesem Fall ist die Amyloidose noch nicht ausgedehnt, und die pathologischen Erscheinungen sind hauptsächlich nur in den. Glomerulis entwickelt, dagegen sind im interstitiellen Gewebe nur wenig Veränderungen zu finden.

Die Glomeruli sind einerseits nur selten zu fibrösen Knäueln vollkommen verödet, andererseits auch nur selten ganz normal. Die meisten Glomeruli zeigen dagegen nur beginnende amyloide Degeneration. Dabei ist die Bowmansche Kapsel wohl erhalten, manchmal mit dem Kapselepithel gut ausgekleidet. In einigen Kapselhöhlen findet sich etwas scholliges Exsudat. Die Amyloidose beginnt am Vas afferens und setzt sich von dort aus mehr oder weniger weit auf einzelnen Schlingen fort. Meist sind nur wenige Schlingen befallen, die sofort durch ihre Verbreiterung und Kernarmut auffallen. In Hämatoxylin-EosinFärbung läßt sich die Membrana propria der Schlingen in einzelnen Knäueln, die quer geschnitten sind, einigermaßen verfolgen. Aber die Beziehung der Membran zu der abgelagerten Amyloide ist schwer zu erkennen. Man bemerkt nur, daß der etwas glänzende lineare Kontur der Membrana propria in der gequollenen Schlingenwand verschwindet. In Methylviolett-Präparaten erkennt man, da es sich hier um Anfangsstadien handelt, sehr deutlich Amyloidstreifchen und -fleckchen in der Wand einzelner Schlingen. Aber in Methylviolett färben sich alle Elemente der Glomeruluswand ziemlich gleichmäßig, so daß die Membrana propria nicht besonders hervortritt. Man gewinnt den Eindruck, daß sie in die Amyloidmasse verschwindet. Nach der van Giesonmethode ist die Membrana propria der Glomerulusschlingen besser zu erkennen. Sie tritt als blaßrötliche Linie hervor. Die amyloiden Teile sind blaß in einem gelbrötlichen Ton gefürbt: Auch hier gelingt es nicht, die Membrana propria durch die amyloiden Partien hindurch zu verfolgen, es sieht vielmehr so aus, als beginne die Membrana propria wieder am Ende der amyloiden Partie. In Reinblaufärbung zeichnen sich die einzelnen amyloiden Schlingen durch ihre diffuse mattblaue Tinktion aus, während die normale Membrana propria eine scharfe blaue Linie darstellt. Hier erkennt man nun an manchen Stellen, daß die blaue Linie der Membrana propria sich mehr nach außen von der amyloiden Substanz in der Schlingenwand verfolgen läßt. An anderen Stellen sieht man sogar. noch, daß die Endothelzellen durch die amyloide Substanz von der Membrana propria fort ins Innere des Capillarlumens verschoben werden. Indessen ist zu erkennen, daß die Linie der Membrana propria immer blasser wird und sich verjüngt. So wechseln die Bilder der amyloiden Schlingenerkrankung. Bald kann man die Linie der Membrana propria noch in das amyloide Gebiet wenigstens eine Strecke weit verfolgen, bald scheint sie im Beginn der Amyloidablagerung ganz zu verschwinden. Bei Bielschowsky-Maresch-Methode nehmen die amyloiden Substanzen graurötliche Färbung an, aber man nimmt keine weiteren Befunde wahr. 
Die Harnkanälchen sind an einzelnen Stellen unverändert. Im Lumen der gewundenen Kanälchen hat sich mehrfach körnige und schollige Masse angesammelt. Nach den Henlesehen Schleifen zu treten hyaline Zylinder auf. An einzelnen Stellen zeigen die Epithelien der gewundenen Kanälchen, nahe dem Übergang in die absteigenden Schleifenschenkel, deutliche Veränderungen, nämlich alle Epithelien zeigen Trübung ihres Protoplasma, das zum Teil viele große hyaline Tröpfchen enthält, ferner Kernverlust, andererseits große Kerne und selbst wohlerhaltene Mitosen. In manchen Tubuli contorti erkennt man noch im Protoplasma des Epithels distinkte, verschieden große kugelige Granula, die in Eosin hellrot erscheinen und veränderten Altmannschen Granulis entsprechen dürften. Sie färbè sich in v. Giesonfärbung glänzend. strohgelb, und in Reinblau-Pikrinsäure-Säurefuchsinfärbung zeichnen sie sich in leuchtend roter Farbe aus. Der Stroma ist an einzelnen Stellen leicht verbreitert durch Zellinfiltrate und im allgemeinen gefäßreich. Diese Zellinfiltrate bestehen aus Lymphocyten und größeren, mit helleren Kernen ausgestatteten Elementen, die den Bindegewebszellen entstammen. Man sieht außerdem kleine lymphocytäre Häufchen um kleine Venen und auch periglomerulär. Hie und da sind die Amyloidablagerungen auch noch in der Wand der Harnkanälchen und der kleineren Arterien anzusehen.

Zusammenfassung. In diesem Fall steht die Amyloidose noch in ihrem Anfangsstadium; sie betrifft nur einzelne Glomerulusschlingen. Gerade darum ist dieser Fall günstig, um die Lagerung der amyloiden Masse, die Beziehung zwischen der Membrana propria und der Amyloidmasse zu studieren. Wir konnten hier einmal konstatieren, was schon Hueter betont hat, daß die Amyloidsubstanz sich nach innen von der Membrana propria ablagert. Weiter sehen wir aber, daß mit der Zusammenschmelzung beider die Affinität der Membrana propria zu den Farbstoffen allmählich abnimmt, bis endlich die Färbung gar verschwindet. Damit können wir sagen, daß die Membrana propria in Kontakt mit der Amyloidmasse leicht ihren Charakter verliert und dadurch die Amyloidmasse zu den Schlingenepithelien in direktere Berührung setzt.

Fall 3. Amyloidniere.

In diesem Fall ist die Amyloidose auch noch wenig vorgeschritten. Von den Glomerulis sind selten einige fibrös sklerosiert und obliteriert, dagegen erscheinen andere fast völlig normal. Die Amyloidablagerung sieht man nur in einigen wenigen Schlingen. In Hämatoxylin-Eosin-Präparaten sind die erkrankten Schlingen hyalin gequollen, kernarm, zeigen aber nicht selten noch erhaltenes Lumen. In dem Lumen bemerkt man gelegentlich Lenkocyten, auch solche, die sich an einer stenosierten Stelle durchquetschen, indem sie sich lang ausziehen. Manchmal sind an der Innenfläche der Capillarwand ganz abgeplattete Kerne zu sehen, die wieder ganz wie Endothelien aussehen. Oft fehlt hier auch jeder Kernbelag. In v. Giesonfärbung tritt die Linie der Membrana propria in ihrem geschwungenen Kontur und leichten Glanz in blaßrötlicher Farbe ziemlich gut hervor. Die amyloide Substanz ist rosig gelb. An einigen Stellen läßt sich feststellen, wie die Membrana propria ohne selbst deutlich verändert zu sein an der Basis des amyloiden Capillarringes vorbeiläuft. Dann wird sie aber stellenweise ganz unsichtbar, verschwindet in der Amyloidmasse. In Reinblau-PikrinsäureSäurefuchsinfärbung ist das Bild viel prägnanter. An den Glomerulis ist dieBowmansche Membran lebhaft blau, die Membrana propria der Schlingen ebenfalls 
deutlich blau, aber ein wenig blasser und das Amyloid selbst mattgraublau. An den einzelnen amyloiden Schlingen zeigt sich, daß die Ablagerung der amyloiden Substanz oft mit dem Verschwinden der blauen Linie der Membrana propria einhergeht. Manchmal erscheint die Membrana propria im Beginn der amyloiden Stellen geradezu wie abgebrochen. In Bielschowsky-Maresch-Methode ist die Membrana propria wieder nicht scharf dargestellt. Man sieht hie und da nur Stücke von schwarzen Linien. Oft finden sie sich gerade am Eintritt der Vasa afferentia unter der amyloiden Substanz. Da, wo die Glomerulusschlinge von Amyloidose betroffen ist, fehlt oft jede Schwarzfärbung, selbst wenn an den anderen Schlingen desselben Glomerulus einige schwarze feine Linien sichtbar sind. Nach diesen Bildern kann also ebenfalls angenommen werden, daß die Membrana propria in der amyloiden Ablagerung schließlich verschwindet.

In den gewundenen Harnkanälchen ist das Epithel zum Teil körnig gegen das Lumen zerfallen. Ein großer Teil der Epithelien hat keine Kerne mehr oder nur noch schattenhafte Reste von Kernstückchen (Chromatolyse). Da die anderen sehr wohl konserviert aussehen, ist anzunehmen, daß der Schwund der Kerne nicht postmortal ist. Die Schleifen der Henleschen Schenkel und die Sammelröhre zeigen ein wohlerhaltenes Epithel. Auch finden sich in den Schleifenkanälchen hie und da hyaline Zylinder, stellenweise bis 5 in einem Gesichtsfeld. In v. Gieson-, Reinblau- und Bielschowsky-Mareschfärbung sind auch die Membrana propriae der Kanälchen ziemlich scharf umschrieben, deutlich gefärbt, wenn auch in der letzten Färbung nicht immer kontinuierlich. Das bindegewebige Stroma ist in der Rinde hie und da etwas verbreitert, besitzt eine leichte Infiltration von lymphoiden Zellen. In $\nabla$. Giesonfärbung sieht man, daß das Bindegewebe übrigens sich um die Glomeruluskapsel verbreitert. Das Stroma in der Marksubstanz ist stark verbreitert, stellenweise hyalin und kernarm. Die Arterienwand ist in ihren größeren Ästen noch zart, frei von Arteriosklerose und zeigt nur Spuren von hyaliner Ablagerung in ihrer Muskulatur, die noch nicht einmal deutliche Amyloidreaktion geben. Die kleinen Arterienäste zeigen, übrigens ebenso wie auch bemerkenswerterweise einige kleine Venen, Amyloid in der Wand, besonders deutlich tritt die Amyloidablagerung in der Wand einzelner Arteriolae rectae hervor.

Zusammenfassung. Da es sich auch in diesem Falle meist nur um einige wenige Glomerulusschlingen handelt, in denen die Amyloidablagerung begonnen hat, ist der Fall wieder zum Studium der Lokalisation der amyloiden Substanz in der Glomeruluswand geeignet. Besonders in diesem Fall konnten wir unter der abgelagerten Amyloidscholle die noch ziemlich gut gefärbte Linie der Membrana propria verfolgen. Danach zeigt sich, daß die Amyloidablagerung in den Glomerulusknäueln gewöhnlich nicht in der Membrana propria selbst, sondern paramembranär stattfindet. Jedoch bleibt dahingestellt, ob bei dem Zustandekommen der Amyloidablagerung nicht besondere feine Veränderungen an der Membrana propria eintreten, die die Amyloidablagerung an Ort und Stelle hervorrufen. Im Laufe des Prozesses wird die Membran bald mitbeteiligt, denn sie verschwindet morphologisch stellenweise in der pathologischen Substanz. Auf die Bedeutung der Membrana propria für das Auftreten der Amyloidmasse in den Knäuelschlingen ist jedenfalls zu achten. 
Fall 4. Amyloidniere.

In diesem Falle fanden sich zugleich Miliartuberkel und käsige Herde in der Niere. Das Organ ist weniger gut konserviert als die beiden vorigen Fälle. Aber vielleicht ist gerade wegen der unvollkommenen Konservierung und einer gewissen kadaverösen Einwirkung das Bild der sog. strukturlosen Membran schärfer gezeichnet als gewöhnlich.

In den Glomeruli sind stets einige Schlingen amyloid, wie die Methylviolettfärbung anzeigt. Die Bowmansche Membran, welche in v. Giesonfärbung rötlich gefärbt ist, tritt deutlich hervor, auch die Membrana propria der Glomerulusschlingen ist ziemlich sinnfällig. Aber in Reinblau-Pikrinsäure-Säurefuchsinfärbung läßt sich die Veränderung der Glomeruli am besten verfolgen, da hier die Membrana propria als scharfe sattblaue Membran vortritt, während die amyloide Substanz mattgraublau ins Gelbliche spielend erscheint. Man stellt fest: da, wo die amyloide Substanz abgelagert ist, ist die blaue Grenzlinie der Membrana propria entweder als äußere Grenze der Glomerulusschlingen sichtbar, oder sie ist unterbrochen. In Bielschowsky-Mareschfärbung zeigt sich nur, daß die amyloiden Schlingen der schwärzlichen Färbung entbehren, wie sie an den nicht amyloiden Schlingen hervortritt.

In den Harnkanälchen haben die Epithelien sich zumeist von der Tunica propria losgelöst. Dabei haben sich die Granula in den Epithelien ganz gut erhalten und sind mit Säurefuchsin gut dargestellt. Die in v. Giesonfärbung rötlich gefärbte Tunica propria tritt als scharfe Linie hervor und hebt sich aufs deutlichste von dem bindegewebigen Stroma ab. In den meisten Arterien der Rinde und des Markes ist auch Amyloid niedergeschlagen.

Zusammenfassung. In diesem. Fall sehen wir dieselben Verhältnisse an den Amyloidmassen und an den Membranae propriae der Glomerulusschlingen wie in den vorigen Fällen. Die Amyloidsubstanz befindet sich immer nach innen von der Membran. Die Membrana propria unter der Amyloidmasse ist bald noch als blasse feine Linie wahrnehmbar, bald verschwunden. Es ist interessant, daß die Membran, auch bei noch geringer Amyloidablagerung, leicht schon verschwinden kann, während sie bei der Argyrie relativ lang, fast bis zur totalen fibrösen Verödung des ganzen Glomerulus ihren Kontur noch wahrnehmen läßt.

Fall 5. Amyloidniere.

Dieser Fall zeigt den höchsten Grad der Amyloidose. Die Niere zeigt im wesentlichen das Bild, der sog. großen weißen Niere.

In den Glomerulis sind die Schlingenwand und das Lumen der Capillaren nur noch an einzelnen Stellen zu sehen. Meist sind die Capillaren in amyloide Bögen ohne Lumen umgewandelt, selten erkennt man noch einen Kern in der Schlinge. An den noch spärlich erhaltenen normalen Capillaren der Glomerulusschlingen ist das Lumen erweitert. In diesem Lumen sind feine rot gefärbte Fibrinfäden und Leukocyten sichtbar, seltener noch bläschenförmige Kerne, die anscheinend. Endothelkerne sind. An den normalen Capillaren der Glomeruli ist die Membrana propria als feine glänzende Linie noch gut zu erkennen, aber an den amyloiden Schlingen verschwindet sie in der amyloiden Substanz. In Reinblau-Pikrinsäure-Säurefuchsinfärbung erscheint die Schlingenwand der erweiterten, nicht amyloiden Teile durch die Membrana propria, die schön blau gefärbt ist, repräsentiert, und außerhalb derselben findet man hie und da nur eine Epithelzelle. Dagegen ist an den amyloiden Schlingen die Linie der Membrana 
propria meist nicht mehr zu sehen, nur in den Grenzpartien geht sie öfters außen an die amyloide Substanz heran, um im Bereiche der amyloiden Teile zu verschwinden. In Bielschowsky-Mareschfärbung zeigt sich an einzelnen Stellen der Glomerulis, daß die Membrana propria schön schwarz gefärbt ist, auch hier ist. im Bereich der amyloiden. Teilevon Schwarzfärbung nicht mehr zu bemerken.

Die Harnkanälchen sind meist erweitert und ihre Epithelien oft abgeplattet. Zylinder sind in den Kanälchen überall in der Rinde und auch in dem Mark reichlich vorhanden. Die amyloide Degeneration betrifft außer den Glomeruli auch die kleinen Arterien der Rinde und die Arteriolae rectae der Pyramiden.

Zusammenfassung. In diesem Fall ist die Amyloidose der Glomeruli so hochgradig, daß die Membrana propria der Knäuelschlingen fast überall verschwindet, An den Schlingen, wo sich die starke Amyloidablagerung vorfindet, ist die Membrana propria nebst Endothelien nicht mehr wahrnehmbar, und die ganze Wand der Schlingen besteht nur aus Amyloidmasse. An den einzelnen Capillaren, die noch von der Amyloidablagerung verschont sind, ist das Lumen erweitert, die Endothelien sind nicht mehr zu finden. An solchen Capillaren bildet die zurückgebliebene Membrana propria die einzige Wand der Capillare. Es ist ohne weiteres klar, daß bei einem solchen Bau der Capillaren fast alle Teile der Funktion erlöschen müssen.

In den vier geschilderten Fällen haben wir einigermaßen die Beziehung zwischen der Amyloidmasse und der Membrana propria der Glomerulusschlingen und besonders das Verschwinden der letzteren bei der Amyloidablagerung beobachtet.

In den folgenden 5 Fällen von Glomerulonephritis wollen wir wieder die Verhältnisse der Membrana propria in den Vordergrund stellen.

Fall 6. Akute Glomerulonephritis in einer leicht cirrhotischen Niere.

Auf den ersten Blick erkennt man in diesem Fall, daß es sich um eine frische Glomerulonephritis mit einer alten Nephritis handelt, denn man sieht, außer den. Bildern der frischen Entzündung, der welligen Linie der Nierenoberfläche entsprechende eingezogene Schrumpfungsherdchen in der Rindensubstanz.

In den zwischen den Schrumpfungsbezirken gelegenen Nierenteilen fällt sofort. die Ungleichheit der nicht obliterierten Glomeruli auf, von denen einige sehr großen Umfang haben und in gleicher Zeit durch ihren Kernreichtum imponieren. An solchen Glomerulis sind die Bowmanschen Kapseln meist ausgedehnt, ihre Epi thelien nur streckenweise erhalten. Adhäsionen zwischen Glomerulus und Kapselwand sind noch nicht vorhanden. Die Glomerulusschlingen enthalten in ihrem Lumen eine große Zahl von gelapptkernigen Leukocyten, mehrfach daneben rote: Blutkörperchen, zuweilen hyalinhomogene, in Eosin rot gefärbte Masse mit oder ohne Leukocyten. Abgesehen von der Leukocytenmenge wird der Zellreichtum der großen Glomeruli auch dadurch bedingt, daß in seinem Bereich zahlreiche Zellen mit ziemlich vielem Protoplasma und einem großen bläschenförmigen Kern vorhanden sind. Diese Kerne sind heller als Leukocytenkerne, rundlich, elliptisch oder etwas eingebuchtet, nur selten chromatinreich. Diese gewucherten Zellen sind sicher keine Leukocyten, aber die Frage ist schwer zu entscheiden, wieweit es Epithelien, wieweit es Endothelien sind. Doch gewinnt man an den quergeschnittenen Glomerulusschlingen oft den deutlichen Eindruck, daß sie wie Endothel in den Glomerulusschlingen selbst liegen. Hyaline fibrinöse Massen zeigen 
sich in den vielen Glomeruli, aber gewöhnlich nur in einer oder zwei Schlingen. Bisweilen liegt der fibrinöse Streifen nur an einer Capillarseite der Glomerusschlingen, derart, daß hie und da ein platter, endothelartiger Kern durch die fibrinöse Masse emporgehoben wird. In manchen quergetroffenen Schlingen erscheint auch ein roter Ring im Innern der Glomerulusschlingen, der noch ein rotes Blutkörperchen umfassen kann. Dabei ist es nicht leicht zu sagen, ob diese fibrinöse Masse auf oder in der Wand liegt. Sie findet sich öfters gerade nahe dem Eintritt des Vas afferens. An einer Stelle geht sie durch dessen Wand bis zur Innenfläche der Glomeruluskapsel und bildet hier einen etwas halbmondförmigen Belag. Die v. Giesonfärbung zeigt die roten Blutkörperehen in den Glomerulusschlingen besser, die fibrinösen Bildungen in den Glomerulusknäueln erscheinen rötlich braun und, sind, von der Membrana propria schwer abzugrenzen. In ReinblauPikrinsäure-Säurefuchsinfärbung ist die Membrana propria der Glomerulusschlingen außerordentlich deutlich als blaue, zuweilen gekräuselte Linie festzustellen. Die hyaline Fibrinmasse ist in den Glomerulis durch Säurefuchsin leuchtend rot gefärbt, während die Erythrocyten (durch Pikrinsäure) strohgelb erscheinen. Man kann hier erst sehen, wie die rote fibrinöse Masse sich auf der blauen Membran der Schlingenwand aufkittet. Hier sind fibrinöse Masse und Schlingenwand offenbar verschieden gefärbt und lassen sich deutlich gegeneinander unterscheiden. Die Bielschowsky-Mareschmethode gibt keine weiteren Aufschlüsse.

Die Harnkanälchen sind erweitert. Größtenteils den Tubuli contorti entsprechend hat das Epithel stärker granuliertes Protoplasma und schöne bläschenförmige Kerne. Einzelne Epithelien aber sind kernlos, andere zweikernig, manchmal fehlen die Kerne in einer ganzen Seite des Zellkranzes von Epithelien. Zuweilen liegen im Lumen abgestoßene Epithelien, die ganz gut erhalten sind und einzelne gelbbraune Pigmentkörnchen enthalten. In manchen erweiterten KanäIchen findet man etwas scholligen oder fädigen Inhalt, auch wenige hyaline Zylinder, besonders in Pyramiden im Bereiche der Henleschen Schleifen. Das Stroma ist in diesem Teil wenig verbreitert, seine Capillaren sind stark erweitert, mit Blut gefüllt. Die meisten Arterienäste zeigen nur eine leichte Verdickung der Intima, die durch Neubildung von Bindegewebe und elastischen Lamellen hervorgerufen wird.

Von den älteren Prozessen sei hier nur kurz erwähnt, daß in den atrophierten Herden die Zahl der verödeten Glomeruli auffallend klein ist, daß aber das Bindegewebe daselbst mächtig gewuchert erscheint. In diesen Herden besteht eine leichte lymphocytäre Infiltration, sind die Harnkanälchen gröBtenteils atrophiert, nur wenige haben noch ein weites Lumen.

Zusammenfassung. In diesem Falle ist der Vorteil der Reinblau-Pikrinsäure-Säurefuchsin-Färbung für die Untersuchung der Membrana propria besonders einleuchtend, weil die Membran und die hyaline Fibrinmasse in den Capillarschlingen verschieden gefärbt sind, die Membrana propria demnach gerade deutlich hervortritt. Wir können somit konstatieren, daß die homogene Fibrinmasse in den Capillaren sicher auf der Innenseite der Membran vorhanden ist. Die Zusammenschmelzung beider findet sich erst später vor.

Fall 7. Akute embolische Nephritis.

In diesem Fall sind die Veränderungen an den Glomerulis interessant. Während ein Teil der Knäuel kaum alteriert ist, sind im anderen einzelne oder mehrere Knäuelschlingen durch den hyalinen, in Eosin rot gefärbten Inhalt sehr auffallend. Die hyalinen Massen in den. Glomerulusschlingen treiben ihre Lumen 
auf und lassen die Schlingen außerordentlich kernarm erscheinen. Mit Immersion erkennt man (in Hämatoxylin-Eosinpräparaten): 1. Vermehrung der Ieukocyten, 2. blaugefärbte Bakterienmasse in den Schlingen, 3. Erythrocyten in einzelnen Schlingen und dann 4. hyaline Fibrinmasse, die das Schlingenlumen mehr oder weniger ausdehnt. Zuweilen sind solche thrombosierte Schlingen von einem Kreis von Leukocyten umringt. Oft ist zwisehen dem hyalinen Thrombus und der Schlingenwand, wenigstens auf einer Seite noch ein leerer Spalt vorhanden, Oft erscheint die Wandlinie der Sohlingen in toto mäßig verbreitert und hyalin. Auf den Glomerulusschlingen liegt außen mehrfach ein Epithelsaum, der den Kapselraum füllt. An manchen Stellen ist zwischen dem hyalinen Thrombus und der Membrana propria keine Grenze zu erkennen. In v. Giesonfärbung sieht man keine weiteren besonderen Befunde. In Reinblau-Pikrinsäure-Säurefuchsinfärbung sind die Membranae propriae der Glomerulussehlingen als blane Linie deutlich siehtbar. Hier sind die hyalinen Thromben durch Säurefuchsin leuchtend rot gefärbt und grenzen sich gut von der Membrana propria ab. Nach der Bielschowsky - Mareseh methode ist in den Glomerulis die Membrana propria sehr wenig gesehwärzt, er scheint nicht deutlich gefärbt, viel mehr die Kerne in den Glomerulis. Aber die Thromben in den Glomerulusschlingen sind gleichmäBig feinkörnig, bläulich schwarz gefärbt und oft ron der Sohlingenwand getrennt. Dureh Bakterienfärbung haben wir kleinere und gröBere Haufen von Streptokokken im Lumen der einzelnen Glomerulusschlingen und Arterienäste konstatiert.

Was das Stroma angeht, so ist es nicht verbreitert, abgesehen von Stellen, wo kleinzellige Infiltrate die Kanälchen auseinander drängen. Diese Infiltrate werden durch Leukocyten, selten durch Lymphocyten und besonders durch Plasmazellen gebildet, schließen manchmal auch Erythrocyten ein und bilden verstreute rundliche Herdchen, die man mit der Lupe gut übersehen kann. Sie liegen vornehmlich in den tieferen Schichten der Rinde, stellenweise auch bis hart an die Oberfläehe hinauf. Sie schließen sich gern an Arterienästchen an, die kleine hyaline Thromben enthalten, oft mit Leukocyten untermengt. Diese Pfröpfe treiben bisweilen das Arterienlumen spindelförmig auf. Manchmal sind die Arterienästchen, welche zu diesen embolischen Herdchen gehören, stark dilatiert und von einem Embolus aus hyalinem Fibrin ganz ausgestopft. Gelegentlich sind die in den Herdohen eingeschlossenen Arterien gerade an der Stelle des mikroskopischen Durchschnittes nicht verschlossen. Die im Bereich dieser erkrankten Arterien gelegenen Harnkanälchen könmen unverändert oder streckenweise nekrotisch sein.

Zusammenfassung. Man kann hier feststellen, daß infolge embolischer, mehr herdförmiger Nephritis, die zum Teil zu einer kontinuierlichen Reihe von Herden führt, eine ziemlich ausgedehnte Glomerulonephritis eingetreten, ist. Die Affektion spricht sich in einer Anhäufung von Leukozyten und hyalinfibrinösen Thrombenmassen in den kleinen Arterienästchen und in den Glomerulusschlingen aus. Die Membrana propria ist oft mit der Oberfläche des hyalinen Fibrins verbacken, aber meist noch selbst an der Wandverbreiterung und Lumenverengerung wenig beteiligt. Indessen finden sich Glomeruli, in denen die Membrana propria durch die hyaline Fibrinmasse etwas glasig gequollen erscheint. Man $\mathrm{m} u ß$ dabei an beginnende fibrinoide Nekrose denken. Doch ist der Prozeß noch jung.

Fall 8. Alte Glomerulonephritis.

In diesem Fall sind die pathologischen Prozesse an den Glomerulis ziemlich vorgeschritten. Die Glomerulusknäuel sind auf den ersten Blick manchmal schwer

Virchows Archiv. Bd. 234. 
wieder zu erkennen. Die Schlingen sind vielfaeh von der hyalinfibrinösen Masse, die in Eosin rot gefärbt ist, erfüllt und deformiert. Sie sind oft an die Kapselwand angewachsen. In den Kapselräumen liegen stellenweise sehr große gewucherte Epithelien mit großen bläschenförmigen Kernen, die größere Kernkörperchen enthalten. Auch zwischen den ziemlich kernarmen Glomerulusschlingen fallen große Epithelien mit großem bläschenförmigen Kern auf, während in den Glomerulusschlingen selbst hie und da sehr platte, auch chromatinreiche Kerne wie ein auskleidendes Endothel zutage treten. Das Lumen der Schlinge ist teils eng, teils weit, und an diesen weiteren Schlingen zeigt sich gelegentlich ein Fibrinring, der das Lumen der quergeschnittenen Schlinge umkreist. Um die Glomeruli ist stellen. weise spindelzellreiches Bindegewebe entwickelt, das in fast konzentrischer Lage in die Glomeruli selbst eindringt, so daß bindegewebige Streifen und hyalin-fibrinöse Masse alternieren. In $\nabla$. Giesonfärbung sieht man einzelne Schlingen deutlich, die noch mit Erythrocyten gefüllt sind. Das neue Bindegewebe ist durch rote Färbung markiert. Aber die Orientierung in den Glomerulis selbst ist ersehwert, namentlich durch die oft beträchtliche Zahl der gewucherten Epithelien auf und zwischen den Glomerulusschlingen. Die Membrana propria tritt an den veränderten Schlingen nur hie und da als rötliche Linie hervor. Selten erkennt man sie noch in den stark erweiterten Schlingen neben dem fibrinösen Material, das braungelb gefärbt ist. In Reinblau-Pikrinsäure-Säurefuchsinfärbung ist die Membrana propria noch deutlicher. Man unterscheidet hier deutlich das feuerrote Fibrin und das orangenfarbige Erythrocyten in den Knäuelschlingen, die von der blauen Linie der Membrana propria oft deutlich begrenzt werden. Auch da, wo das Fibrin mit der Kapsel verschmolzen ist, ist es ganz deutlich zu umschreiben. Stellenweise ist die Membrana propria in dem verbacknen Fibrin nicht mehr wiederzuerkennen. Bielschowsky-Mareschmethode ergibt hier auch keine weiteren Aufsohlüsse.

Die Harnkanälchen sind vielfach mit wohlerhaltenen ausgelaugten Erythrocyten erfüllt. In anderen Kanälchen finden sich nekrotische abgestoßene Epithelien und Leukocyten oder auch fibrinähnliche Zylinder. Das Stroma ist ziemlich stark verbreitert und ziemlich zellreich. Diese Zellen sind teils Plasmazellen, teils Lymphocyten, die sich besonders im Umfang der stark verödeten Glomeruli anhäufen. In den kleinen Arterien ist zuweilen die Media hyalinfibroid ungewandelt und das Epithel im Lumen stellenweise geschwollen.

Zusa m menfass ung. Hier sieht man wieder fibrinöse Gerinnung in den Glomerulusschlingen, die an einzelnen Stellen auch außerhalb der Schlingen vorhanden sind und dann zur Verschmelzung mit der Kapsel führen. An solchen Punkten kann die Membrana propria nicht gut hervortreten oder in die fibrinöse Masse aufgehen.

Fall 9. Nephritis glomerulo-tubulo-interstitialis.

In diesem Fall sind außer den Glomerulis Harnkanälchen und Stroma auch erkrankt. Die Glomeruli sind groß, kernreich und nur an einzelnen Stellen an der Kapsel adhärent. Das Kapselepithel ist stellenweise geschwollen, stellenweise abgeplattet. Die Glomerulusschlingen enthalten noch vielfach Blutelemente mit mäßiger Zunahme der Leukocyten. Ihr Kernreichtum ist aber vornehmlich durch Wucherung von Epithelien und Endothelien bedingt. Das fibrinöse Material in den einzelnen Schlingen ist in v. Giesonfärbung schön orangenrot gefärbt. Man sieht es auch getrennt, vom Glomervilus auf der Innenfläche der Kapsel sich ausscheiden. Hier bildet das Fibrin ein Maschenwerk, das auf dem Kapselepithel ruht, aber auch zwischen ihm bis auf die Bowmansche Membran eingreift, die in v. Giesonfärbung wieder rot gefärbt ist. In den Glomerulis sind die Membranae 
propriae der Knäuelschlingen scharf markiert. Auf ihnen ist das außen festsitzende Epithel mit geschwollenen, bläschenförmigen Kernen deutlich kenntlich. Im Schlingenlumen sieht man mehrfach Leukocyten, einzelne Erythrocyten und auch Zellen, die als Endothelien angesprochen werden können und sich zuweilen abgelöst haben. In Reinblau-Pikrinsäure-Säurefuchsinfärbung ist die Membrana propria noch sehr scharf gezeichnet, und die oben erwähnten Verhältnisse der Membrana propria und der fibrinoiden Masse lassen sich allerwärts deutlich wahrnehmen.

Die Harnkanälchen der Rinde sind stark erkrankt. Sie sind fast alle erweitert, ihr Lumen selten leer, meist mit körnigen Massen, Erythrocyten, Leukocyten, hyalinen Schollen oder endlich mit Gemischen von diesen Elementen erfüllt. Ihre Epithelkerne sind stellenweise defekt, stellenweise deutlich gewuchert. Im Stroma finden sich vielfach Anhäufungen von Lymphocyten und. Plasmazellen.

Zusammenfassung. Die wesentliche Veränderung betrifft in diesem Fall Kanälchen und Stroma. Die Glomeruli zeigen auch Veränderungen, aber die Membrana propria ist fast intakt und zugleich der Niederschlag fibrinösen Materials in den Schlingen recht gering.

Fall 10. Akute hämorrhagische Glomerulonephritis mit ausgedehnter Thrombosierung der Glomerulusschlingen in der "Schwangerschaftsnephritis" ohne Eklampsie.

In diesem Fall sind fast alle Glomeruli schwer erkrankt. Sie sind sehr groß. Das Lumen ihrer Schlingen ist stark erweitert und strotzend mit Blut erfüllt. In den Kapselräumen sieht man auch ausgetretene Erythrocyten. In den Glomerulusschlingen sind außerdem viel fibrinöse Massen und zahlreiche Leukocyten sichtbar. Die fibrinösen Massen schlagen sich in ihnen teils als hohlzylindrische Wandbeläge der Schlingen, teils als solide Ausfüllung des Lumens nieder. Da, wo noch ein freies Lumen übrig bleibt, ist es von Blutzellen oder von einem Endothelkerne eingenommen. Die hyalinen Fibrinmassen setzen sich oft auf das Vas afferens und de etwas zurückliegende Arterienstrecke fort, dabei liegen diese Massen zum Teil in der Wand, zum Teil im Lumen. Die Endothelien sowie auch die Epithelien im Glomerulus sind im allgemeinen spärlich, ihre noch erhaltenen Kerne ziemlich dunkel gefärbt. Jedenfalls gibt es keine Wucherung derselben. In v. Giesonfärbung ist die rote Linie der Membrana propria ziemlich deutlich von den hyalinen fibrinösen Massen zu trennen. Da, wo durch die oberflächlichen Schlingen Diapedese stattfindet, sieht man die Linie der Membrana propria als scharfe Grenze zwischen den intra- und extravasculären Erythrocyten. An dem extravasierten Blut kann es zur Fibrinausscheidung kommen, die sich auf der Membrana propria aufbaut. In Reinblau-Pikrinsäure-Säurefuchsinfärbung sieht man den ersten. Beginn der intracapillaren Fibrinausscheidungen im Glomerulus noch besser. Sie beginnt damit, daß sich auf der blauen Linie der Membrana propria kleine hyaline Fibrinschollen ablagern, die dann konfluieren und dann Bänder oder Ringe bilden, die Auflagerung beginnt also diskontinuierlich. Selten ziehen die Fibrinnetze von Anfang an durch das Lumen der Capillaren.

In den Harnkanälchen ist auch das Epithel in ausgedehnter Weise nekrotisch. In ihrem Lumen ist viel Blut ausgetreten. Die größeren Arterien sind frei.

$Z$ usammenfassung. In diesem Fall sehen wir verschiedene Bilder der Erkrankung. In den Harnkanälchen tritt die Alteration in Form von Nekrose auf. Von seltener Schwere und Ausdehnung ist die Erkrankung der Glomeruli und Vasa afferentia: durch mächtige fibrinöse Ausscheidung in der Wand und auch im Lumen der kleinen 
Arterien, Verstopfung der Glomerulusschlingen, durch hochgradige ausgedehnte Blutungen in den Kapselräumen und in den Kanälchen, bedingt durch Verschluß des Schlingenlumen und durch Alteration der Wand. Interessant sind dabei die Vorgänge der Fibrinausscheidung in den Glomerulusschlingen, die zur Beobachtung kamen. Sie beginnt zuerst auf der Innenfläche der Membrana propria diskontinuierlich, und nicht von Anfang an als gleichmäßiger Belag.

\section{Schluib betrachtungen.}

Wir haben verschiedene pathologische Nierenprozesse gewählt, um das Verhalten der Membrana propria bei ihnen zu verfolgen. Wie eingangs erwähnt ist, sind solche Studien nur durch besondere geeigneten Färbungen möglich, die dieses zarte Häutchen schärfer hervortreten lassen. Mit den gewöhnlichen Färbmethoden kommt diese feine Membran so wenig zum Vorschein, daß man sie eben geradezu übersieht. Das ist, wie ich glaube, gerade die Hauptursache davon, daß die Studien der Membrana propria der Glomerulusschlingen in vielen Fällen bisher vernachlässigt werden. Für diesen Zweck haben wir Reinblau angewendet und mit ihm ausgezeichnete Resultate erhalten.

Wir haben zunächst einen Zustand gewählt, in dem sich eine abnorme Pigmentierung im Gegensatz zu anderen pathologischen Pigmenten vorzüglich in dem Glomeruli offenbart. Die Silberfärbung durch die auf alimentärem Wege eingeführten Silberpräparate stellt sich, wie man seit langem weiß, in bestimmten Organen ein und heftet sich an die Grundsubstanzen. Dadurch unterscheidet sie sich von den Silberniederschlägen, die nach intravenöser Kolloidalsilbereinspritzung zustandekommen und die sich besonders an den Zellen des Blutfilterapparates (Leber, Milz, Knochenmark) beobachten lassen. Von der Argyrie ist nun schon für einzelne Teile so für die Plexus chorioidei nachgewiesen, daß die Silberkörnchen sich in einer Grenzmembran zwischen Epithel und Bindegewebe ansammeln (Askanazy). Es war daher interessant, die Stelle festzustellen, wo das Silberpigment in den Glomerulusschlingen anzutreffen ist. Wie oben eingehend geschildert ist, haften die Körnchen alle an der Membrana propria, in der sie gleichmäßig fein verstäubt sind. Sie nehmen an den Schicksalen dieser Membran teil. Wenn in unserem Falle einzelne Glomerulusknäuel durch eindringendes Bindegewebe komprimiert, endlich zu einem lumenlosen Klumpen zusammengeschweißt werden, so läßt sich jede Phase an der versilberten Membrana propria besser verfolgen. Je kleiner der Glomerulus, um so tiefer seine Schwarzfärbung trotz gleichbleibender Silbermenge. Auch die Verlegung der Lumina der Schlingen wird durch die Pigmentierung besser kenntlich. Weder Endothelnoch Epithelzelle enthalten das Pigment. Diese spezifische Ablagerung 
der Silberkörnchen in der Membrana propria ist wohl die beste Demonstration für ihre pathologische Bedeutung, da sie ganz elektiv erfolgt, ohne Beteiligung anderer Glomeruluselemente, wenn von der geringen Ablagerung in der Bowmanschen Membran abgesehen wird. Ebenso ist es interessant, das Seitenstück für den Vorgang in der freilich schwächeren Silberablagerung in den Tunicae propriae der Harnkanälehen in der Pyramidensubstanz zu finden. Die Aufnahme des Silbers aus dem vorüberströmenden Blut in den Glomerulusschlingen kann man sich chemisch oder wohl besser physikalisch-chemisch vorstellen. Sie illustriert jedenfalls die Tatsache der elektiven Beteiligung der Membrana propria an der Ausscheidung pathologischer, körperfremder Stoffe aus dem Blute, wenn diese Stoffe auch in der Wand abgefangen und lange festgehalten werden.

Als zweites Objekt wählten wir einen Prozeß, bei dem sich abnorme chemische Produkte, die im Körper entstehen und sich unter anderem in der Niere niederschlagen, vorfinden. In diesen Fällen von, Amyloidnieren haben wir die Membrana propria von den anderen Glomerulusbestandteilen gut zu trennen vermocht. Unsere Befunde in der Amyloidose der Glomeruli stimmen zum Teil mit den Feststellungen von Hueter überein. Die Amyloidmasse lagert sich in der Tat in den Glomerulis fast immer nach innen von der Membrana propria ab. Aber dieses Stadium hat keinen Bestand. Im Anfang konnten wir die amyloide Masse getrennt von der blaugefärbten Membran verfolgen, aber mit der Zeit wird die Amyloidzone verbreitert und damit die Membrana propria in Mitleidenschaft gezogen. Zwar kann das Amyloid nach innen ausweichen und das Lumen der Capillare verengern, endlich ganz verlegen. Allein die Membran bleibt nur im Anfangsstadium anscheinend gut erhalten, durch deutliche Färbung noch gut nachweisbar, aber dann verliert sie bei ihrer Verschmelzung mit der Amyloidmasse ihre Färbungsfähigkeit nach und nach, bis sie endlich nicht mehr erkennbar ist. Sie scheint in der Amyloidmasse zu verschwinden, dann kann man sie nicht mehr in der Amyloidmasse wiederfinden. Danach spielt die Membrana propria auch bei der Amyloidose des Glomerulus ihre Rolle. Vielleicht ist sie dabei nur passiv beteiligt. Da wir aber wissen, welche intimen Beziehungen zwischen Amyloid und Grundsubstanz obwalten, ist es wohl möglich, daß zwischen den Substanzen der Membrana propria und des Amyloides intimere Beziehungen bestehen.

Endlich kommen wir zu der Glomerulonephritis, wo alle Elemente der Knäuel bei der gestörten Funktion in Betracht zu ziehen sind. Unsere 5 Fälle sind verschiedener Art, teils ganz frisch, teils frische Prozesse bei alter Nephritis, teils ältere Stadien der Glomerulusaffektion. Am wichtigsten für unser Thema ist die Beziehung zwischen 
den Fibrinausscheidungen in den Schlingen und der Membrana propria. Die Massen des hyalinen Fibrins welchseln. Sie scheiden sich auch außen im Raum zwischen Kapsel und Glomerulusoberfläche $a b$, wobei denn ebenso wie bei den Blutungen per diapedesin die Membrana propria die Grenzlinie markierte. Bei der Fibrinausscheidung in den Schlingen war die Membrana propria auch in der Regel die Basis, auf der sich das Fibrin ablagerte. Sie setzt sich zuerst auf der Membran $a b$, und zwar anfangs in Form von feinen Schollen, die erst später mit einander konfluieren. Aber die Färbung der Membrana propria bleibt länger als in den Amyloidnieren intakt, und selbst wenn das Schlingenlumen durch abgelagerte Fibrinmassen ganz verstopft oder noch durch die Vergrößerung derselben ziemlich stark dilatiert wird, kann man sie deutlich von den Fibrinmassen unterscheiden. Aber dann kommt der Augenblick, wo das fibrinöse Material auf die Wand selbst übergreift, ja durch sie hindurchzieht. Die Färbung der Membrana propria blaßt bei ihrer. Verschmelzung mit den fibrinösen Massen allmählich $a b$, bis sie endlich verschwindet und die Unterscheidung zwischen ihnen nicht mehr möglich ist. Also sehen wir hier die Membrana propria ein ähnliches Schicksal erleiden wie bei der Amyloidose.

Bei Glomerulonephritis ergibt sich aber auch eine besondere Frage, nämlich die nach der fibrinoiden Umwandlung der Membrana propria. Wenn sich zeigt, daß die fibrinoide Nekrose der bindegewebigen Grundsubstanz eintritt, sobald das Bindegewebe seine Vitalität verliert, kann man dann annehmen, daß die Membrana propria dann selbst in fibrinoides Material aufgeht, wenn die Capillarwand vital schwer geschädiist? Die Antwort hängt davon $a b$, ob eine solche Lumen und Wand der Schlingen betreffende sog. ,hyaline Degeneration" mit Schwund der Kerne einhergeht. Schon Löhlein hat Fälle abgebildet, wo an den kranken Glomeruli neben den kernreichen Schlingen homogenisierte Partien vorhanden sind, die sich durch Kernarmut auszeichnen. In unserem Falle von akuter hämorrhagischer ,Schwangerschaftsnephritis" waren auffallend wenig Kerne in den schwer erkranken Glomeruli zu sehen.

Bei dem Zugrundegehen der Membrana propria in den oben geschilderten drei pathologischen Vorgängen gibt es nun doch gewisse Unterschiede in der Art und in der Zeit ihres Untergangs. Bei Amyloidose und Glomerulonephritis geht sie relativ früh unter, während sie bei Argyrie eigentlich mehr verdeckt als zerstört wird. Fast bis zur totalen fibrösen Verödung der Glomeruli lassen sich hier ihre geschlungenen Konturen noch wahrnehmen. Diese Unterschiede bei pathologischen Einwirkungen sind in erster Linie in den Verschiedenheiten des Wesens der betreffenden Erkrankungen begründet. So liegt bei Amyloidose oder Glomerulonephritis das Wesen der Erkran- 
kung hauptsächlich in den chemischen Veränderungen der Glomerulusschlingen selbst, während bei Argyrie es sich vielmehr um die Vorgänge wie bei einem physikalischen Proze $B$ handelt.

Schon nach diesen Kenntnissen ist es ohne weiteres klar, daB auch bei andersartigen, ja bei allen Erkrankungen der Glomeruli das Verhalten der Membrana propria der Glomerulusschlingen genaue Beachtung verdient. Ihr Schicksal kann sich nach den Umständen verschieden gestalten: Die Membran kann mechanisch zusammengepreBt, sie kann physikalisch und auch chemisch verändert werden. Um diese Verhältnisse weiter klarzustellen, ist es nötig, Untersuchungen an noch umfassenderem Material bei verschiedenen Glomeruluserkrankungen auszuführen. Wir selbst werden später Gelegenheit haben, auf diese Fragen zurückzukommen. Wie dürfte man auch eine morphologisch in ihren Schicksalen zu verfolgende Membran in einem mikroskopischen Organbezirk vernachlässigen, dessen Funktion in einer Art Filtration besteht!

\section{Zusammenfassung.}

1. Die Membrana propria der Glomerulusschlingen, die ehedem etwas berücksichtigt wurde, aber in den letzten Jahren mehr in Vergessenheit geriet, ist wirklich vorhanden.

2. Die Reinblaufärbung ist zweckmäßig für ihre Darstellung.

3. Diese Membran zeigt eine elektive Attraktion für gewisse Substanzen im Blut (Argyrie).

4. Bei Amyloidose lagert sich Amyloidmasse zunächst nach innen von der Membrana propria, dann in ihr selbst ab, und diese verschwindet schließlich in jener.

5. Bei Glomerulonephritis sind die Bilder der Fibrinabscheidung im allgemeinen ähnlich wie bei Amyloidose, nur läßt sich die Membrana propria hier lange abgrenzen.

6. Die Membrana propria der Glomerulusschlingen sollte in der Nierenpathologie noch mehr berücksichtigt werden.

\section{Literaturverzeiehnis.}

Aschoff, Pathologische Anatomie 1920. - Baehr, Über experimentelle Glomerulonephritis. Zieglers Beiträge z. allg. Path. u. pathol. Anat. 55, Heft 3. 1913. - v. Ebner, Koellikers Handbuch der Gewebslehre. 3. 1902. - Fahr, Über herdförmige Glomerulonephritis. Virchows Arehiv 225, Heft 1. 1918. Goldschmid, E., Lebercirrhose und Kalkinfarkt der Nierenpyramiden. Zieglers Beiträge z. allg. Path. u. pathol. Anat. 56, Heft 1. 1913. - Gross, Frische Glomerulonephritis. Zieglers Beiträge z. allg. Path. u. pathol. Anat. 65, Heft 3. 1919. — v. Hansemann, Zur pathologischen Anatomie der Malpighischen Körperchen. 
72 D. Ohmori : Bedeutung der Membrana propria der Glomerulusschlingen usw.

Virchows Arehiv 110, Heft 1. 1887. - Herxheimer, Nierenstudien II. Über Anfangsstadien der Glomerulonephritis. Zieglers Beiträge z. allg. Path. u. pathol. Anat. 64, Heft 3. 1918. - Hueter, Über Amyloid der Glomeruli. Centralbl. f. allg. Pathol. u. pathol. Anat. 19, Nr. 23. 1908. - Löhlein, Entzündliche Veränderungen der Glomeruli der menschlichen Niere. Arbeiten aus dem Pathol. Institut zu Leipzig 1. 1907. - Löhlein, Über Schrumpfniere. Zieglers Beiträge z. allg. Path. u. pathol. Anat. 63, Heft 2. 1917. - Schultze, Stöhrs Histologie 1919. 